\title{
Uterine rupture disguised by urinary retention following a second trimester induced abortion: a case report
}

\author{
Qiaoying Jiang ${ }^{1+}$, Liwei Yang ${ }^{1,3^{*}+}$, Charles Ashley², Erin E Medlin², David M Kushner ${ }^{2}$ and Yanmei Zheng ${ }^{1}$
}

\begin{abstract}
Background: Uterine rupture classically presents with severe abdominal pain, loss of fetal station, vaginal bleeding, and shock.

Case presentation: We present a case of uterine rupture presenting as significant urinary retention that occurred following a second trimester abortion induced with mifepristone and misoprostol. Uterine rupture was discovered unexpectedly on diagnostic laparoscopy. The uterine rupture was contained by dense adhesions between the omentum and bladder with the previous uterine cesarean hysterotomy scar.

Conclusion: This case highlights the difficulties in diagnosis of abnormal placentation and an unusual presentation of uterine rupture. This case was managed successfully laparoscopically.
\end{abstract}

Keywords: Uterine rupture, Urine retention, Misoprostol, Mifepristone, Laparoscopy

\section{Background}

Second trimester pregnancy termination is a common gynecologic procedure and may be performed by surgical evacuation or induction of labor. Both surgical evacuation and induction of labor are safe and have rare complications including infection, blood loss, uterine rupture or perforation. Misoprostol is a synthetic prostaglandin E1 analogue which is widely used for induction of labor and is an effective agent for second trimester abortion [1]. Retrospective analysis has shown that mifepristone combined with a prostaglandin analogue in second trimester abortion is not associated with higher morbidity in women with a prior cesarean section [2]. A rare complication of second trimester induction of labor with a previous uterine scar is uterine rupture, which most commonly presents with abdominal pain, loss of fetal station, vaginal bleeding, and shock [3]. Here, we present an interesting case of uterine rupture during a

\footnotetext{
* Correspondence: yanglw1967@163.com

${ }^{\dagger}$ Equal contributors

'Department of Obstetrics and Gynecology, Zhejiang Provincial People's

Hospital, Hangzhou, Zhejiang, People's Republic of China

${ }^{3} Z$ hejiang Provincial People's Hospital, NO.158 Shangtang Road, Hangzhou,

Zhejiang Province 310014, China

Full list of author information is available at the end of the article
}

second trimester abortion after oral administration of mifepristone and misoprostol. The patient presented with idiopathic fever and urinary retention, and the uterine rupture was diagnosed unexpectedly during exploratory laparoscopy.

\section{Case presentation}

A 31-year-old multigravid woman with a history of a prior low transverse cesarean delivery presented to a family planning clinic at 16 weeks gestation for elective termination of pregnancy. Her obstetric history was significant for a prior cesarean section performed six years prior. Her past medical and surgical history was otherwise unremarkable. A transabdominal ultrasound performed on the day of admission confirmed a fetus of 16 week gestation with an anterior placenta. In order to further delineate the relationship between the uterine scar and the placenta, an abdominal MRI was performed. The MRI demonstrated an unclear uterine scar near the placental implantation site. The patient was counseled on risks associated with abnormal placentation and was offered uterine artery embolization, dilation and evacuation and hysterotomy. The patient, however, decided to proceed with induction of labor for termination. The termination of her pregnancy was approved 
by the ethics committee of Zhejiang Provincial People's Hospital. A written informed consent was obtained from this patient before the procedure.

Termination was performed with a loading dose of $50 \mathrm{mg}$ of oral mifepristone (RU486) followed by $25 \mathrm{mg}$ administered every 12 hours until a total of $300 \mathrm{mg}$ had been administered. Following completion of mifepristone, misoprostol was administered at a dose of $200 \mathrm{ug}$ orally every hour. The patient received a total of $1200 \mathrm{ug}$ of misoprostol. She had an unremarkable vaginal delivery with spontaneous delivery of the placenta. Vaginal bleeding was minimal. Four hours after the procedure the patient developed a fever of $38.5^{\circ} \mathrm{C}$, but denied further symptoms. The patient's temperature resolved spontaneously by the next morning without intervention. On post procedure day 1 , the patient complained of abdominal distention and dysuria. A urethral catheter was inserted and $800 \mathrm{ml}$ of clear urine was drained. A pelvic ultrasound was performed and demonstrated a $32 \times 25 \mathrm{~mm}$ abnormal radiodensity at the anterior uterine wall with an unclear boundary at the anterior cervical wall, concerning for retained products of conception versus placenta accreta. Methotrexate was administered. Given the idiopathic urinary retention and abnormal findings on ultrasound, laparoscopic exploration was performed to evaluate for placental growth into in bladder.

At the time of diagnostic laparoscopy, no hemoperitoneum was noted; however, there were significant adhesions between the omentum and anterior abdominal wall. Unexpectedly, a uterine rupture at the lower uterine segment was observed when the pelvic organs were restored to their normal anatomy. There was a $1.5 \mathrm{~cm}$ length anterior wall uterine rupture along the left portion of the previous scar (Figure 1). Moreover, a portion of the placenta was found to be adherent to the uterus at the site of the rupture when the omentum was removed (Figure 2). The bladder was firmly adherent to

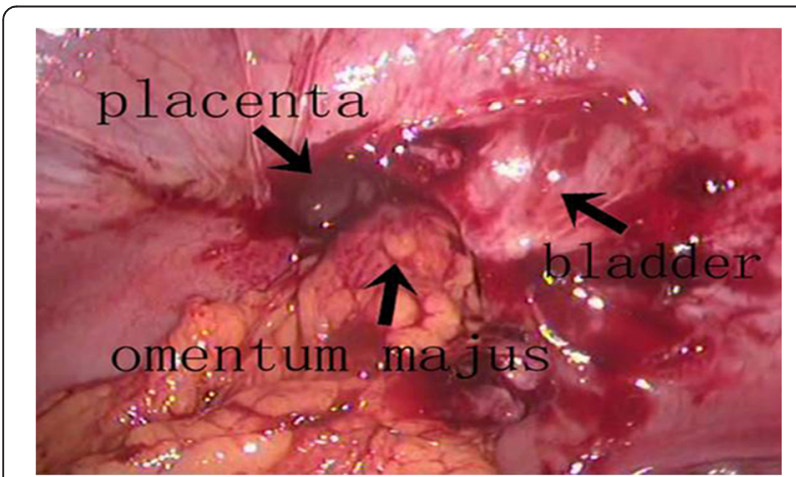

Figure 1 A length of $1.5 \mathrm{~cm}$ anterior wall uterine rupture along the left previous scar was found. The rupture was filled with small amounts of placenta and omentum majus.

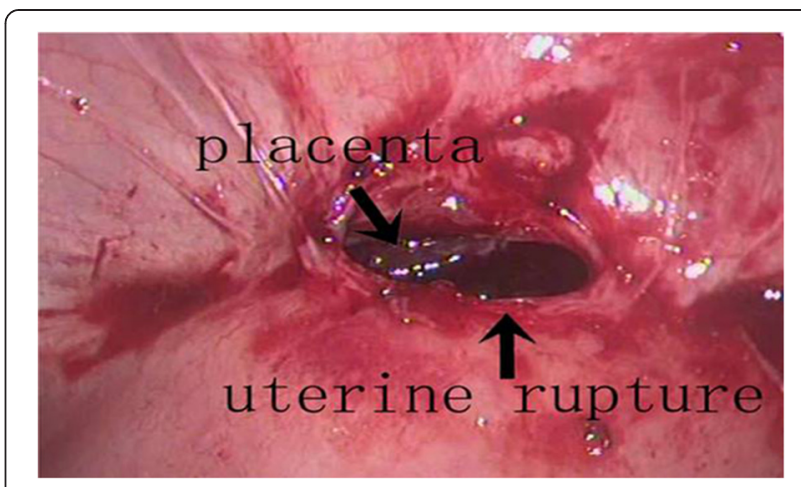

Figure $2 \mathrm{~A}$ patch of placenta adhered to the rupture and lower uterine segment was observed when the omentum majus removed.

the uterine scar and rupture, and urology was consulted to assist in separating the bladder from the uterus. Dilatation and curettage was performed under direct laparoscopic visualization and the specimens were confirmed to be placenta by postoperative pathology examination (Figure 3). An ultrasound was performed to ensure no residual placental tissue was present. The uterine dehiscence was repaired laparoscopically. The entire procedure was performed using minimally invasive techniques.

\section{Discussion}

Misoprostol has been widely used for medical abortion. Mifepristone has also been reported to be relatively safe and effective for use in the second trimester $[4,5]$. According to the recent clinical guidelines published by the Society of Family Planning (\#2013-4), misoprostol is widely used as an off-label alternative or adjunct to osmotic dilators prior to dilation and evacuation in women desiring a second trimester pregnancy termination [6]. It is also safely used for labor-induction in mid second trimester abortions, even in women with prior uterine surgery [7]. A recent systematic review reported that the

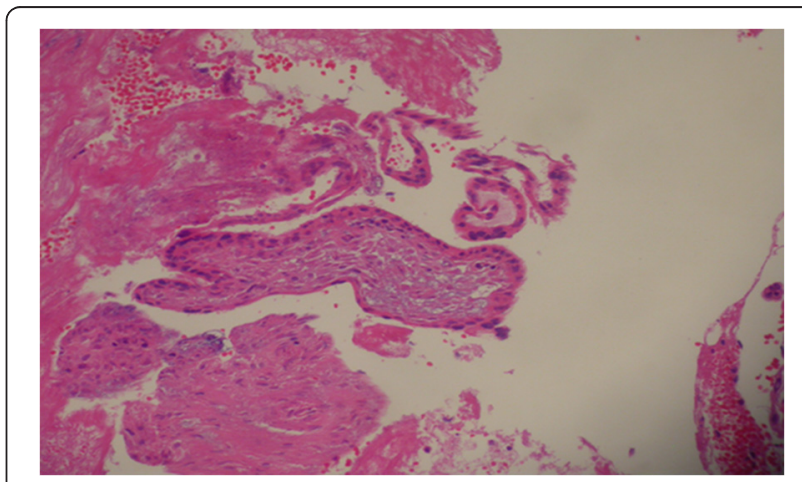

Figure 3 Specimens sent for pathological examination were confirmed to be placenta. 
risk of uterine rupture in these women was less than $0.3 \%$ [3].

Uterine rupture is a rare but serious complication of second trimester induction of labor. Severe lower abdominal pain and shock caused by intraabdominal hemorrhage is the classic clinical presentation [8]. This patient, however, presented insidiously with urinary retention. The patient's vital signs were clinically stable, and she reported no symptoms of abdominal pain. The ultrasound and MRI performed before the abortion raised concerns for abnormal placentation. The presentation of urinary retention raised concern for abnormal placentation with potential bladder involvement, although the patient had no evidence of hematuria, Methotrexate was administered timely to induce necrosis of trophocytes. Laparoscopic exploration was performed before dilatation and curettage to evaluate for bladder involvement. The uterine rupture was an unsuspected finding during the operation. The rupture was contained within the vesicouterine space, leaving only a small hole obscured by omental adhesions. Although it is unclear when the uterine scar dehisced, we suspect that this occurred prior to delivery given the dense adhesions, and further developed to uterine rupture with labor. Previous reports have noted similar findings of second trimester uterine rupture in the setting of abnormal placentation. This case was successfully managed laparoscopically. There is only one previous case report has described repair of uterine rupture with laparoscopic techniques [9].

\section{Conclusions}

In summary, this is an unusual case of uterine rupture presenting with a primary complaint of urinary retention. This case highlights the difficulties in diagnosis of abnormal placentation and an unusual presentation of uterine rupture. Following a second trimester abortion, uterine rupture should be included in the differential diagnosis for patients presenting with urinary retention and continued vaginal bleeding. This case was successfully managed with laparoscopy. Minimally invasive surgery can be performed safelyy following a second trimester abortion and should be considered for management of complications following elective second trimester abortion.

\section{Consent}

Written informed consent was obtained from the patient for publication of this case report and any accompanying images. A copy of the written consent is available for review by the editor of this journal.

\section{Competing interests}

The authors declare that they have no competing interests.

\section{Authors' contributions}

LY has performed a major role in the diagnosis, treatment of the patient, collection of the informed consent and the design of the manuscript. QJ took an important part in surgical treatment of the patient, literature search, writing and review the manuscript. CA, EEM and DMK took part in conception of the manuscript, literature search and review. YZ was involved in surgical treatment of the patient and the follow-up of the case. All authors read and approved the final manuscript.

\section{Authors' information}

Liwei Yang is a doctor of the department of Obstetrics and gynecology, graduated from Zhejiang University and achieved master's degree. Papers have been published are listed below:

YANG Liwei, LIN Jun, WU Ruijin, et al. IL-6, mIL-6R and sIL-6R levels in peritoneal fluid of women with endometrios, Zhejiang Medical Journal, 2010,32(2):169-172.

YANG Li-wei,YING Qi-feng,MAO Jia-hui. The urine deoxypyridinoline/creatinine ratio and its relationship with serum estradiol in perimenopausal and menopausal women. The Chinese journal of gerontology, 2009, 28(12):991-993.

YANG Liwei, WU Ruijin, LIN Jun, et al. The role and expression of membrane interleukin 6 receptor in peritoneal fluid of women with endometrios, Chin J Obstet Gynecol, 2008,43(5):368-369.

\section{Acknowledgments}

The authors would like to thank Professor Yaer Lv and Feng Liu for their support in the diagnosis and treatment of the patient.

\section{Author details}

${ }^{1}$ Department of Obstetrics and Gynecology, Zhejiang Provincial People's Hospital, Hangzhou, Zhejiang, People's Republic of China. ${ }^{2}$ Department of Obstetrics and Gynecology, University of Wisconsin School of Medicine and Public Health, Madison, Wisconsin, USA. ${ }^{3}$ Zhejiang Provincial People's Hospital, NO.158 Shangtang Road, Hangzhou, Zhejiang Province 310014, China.

Received: 13 September 2014 Accepted: 17 December 2014 Published online: 22 January 2015

\section{References}

1. Dodd JM, Crowther CA. Misoprostol versus cervagem for the induction of labour to terminate pregnancy in the second and third trimester: a systematic review. Eur J Obstet Gynecol Reprod Biol. 2006;125:3-8.

2. Mazouni C, Provensal M, Porcu G, Guidicelli B, Heckenroth H, Gamerre M, et al. Termination of pregnancy in patients with previous cesarean section. Contraception. 2006;73:244-8.

3. Goyal V. Uterine rupture in second-trimester misoprostol-induced abortion after cesarean delivery: a systematic review. Obstet Gynecol. 2009;113:1117-23.

4. Nguyen TNN, Tara S, Sheila R, Blum J, Nga NT, Minh NT, et al. Mifepristone and misoprostol compared with misoprostol alone for second trimester abortion. Obstet Gynecol. 2011;118:601-8.

5. Dickinson JE, Jennings BG, Doherty DA. Mifepristone and oral, vaginal, or sublingual misoprostol for second-trimester abortion. Obstet Gynecol. 2014;123:1162-8.

6. Fox MC, Krajewski CM. Cervical preparation for second-trimester surgical abortion prior to 20 weeks' gestation: SFP Guideline \#2013-4. Contraception. 2014;89:75-84

7. Frick AC, Drey EA, Diedrich JT, Steinauer JE. Effect of prior cesarean delivery on risk of second-trimester surgical abortion complications. Obstet Gynecol. 2010;115:760-4

8. Sliutz G, Sanani R, Spängler-Wierrani B, Wierrani F. First trimester uterine rupture and scar pregnancy. Med Hypotheses. 2009;73:326-7.

9. Ramirez ER, Hakakha B, Dale K, Khatame M. The role of laparoscopy in the management of second trimester spontaneous uterine rupture. Obstet Gynecol. 2014;123 suppl 1:128S. 